\title{
The effect of task and stimulus variability on habituation of electrodermal and vasomotor reactions
}

\author{
R. L. RAY, J. F. PIROCH, and H. D. KIMMEL \\ University of South Florida, Tampa, Florida 33620
}

\begin{abstract}
The effects of reaction time (RT) task and stimulus variability upon the habituation of skin conductance responses (SCR) and vasomotor responses (VMR) were studied in two experiments. In Experiment 1, subjects who performed an RT response to stimulus onset or who received stimuli which varied in frequency required more trials to reach an SCR habituation criterion than subjects who did not perform the task or receive frequency variability. While the overall VMR magnitude was affected by RT task and frequency variability, no VMR habituation itself was observed. In Experiment 2, the effects of RT task to stimulus offset and stimulus-duration variability were investigated. RT task again produced retardation of SCR habituation. This indicated that the task effect was not simply due to contraction-elicited SCRs, since the task reactions occurred well after the onset-elicited SCRs. Stimulus-duration variability reduced the overall effect of task, and it was hypothesized that manipulations which tend to increase postonset SCR reactivity have a negative effect on the persistence of onset reactivity.
\end{abstract}

Habituation is an important phenomenon, not only because of its status as a simple kind of learning (Thompson \& Spencer, 1966; Thorpe, 1956; Razran, 1971), but also because of its role in recent theoretical formulations regarding conditioning. For example, Kimmel (1973) has proposed that when two reflexes are paired and the second of these is of the homeostatic, negative feedback type, the habituability of the first reflex relative to the second may determine both the nature and ease of acquisition of the conditioning that results. Kozak and Westerman (1966) have made a similar proposal, although they treat habituability as an invariant property of reflex type, while Kimmel has stressed the potential manipulability of habituability. Dykman (1976) has also stressed the role of habituability in a recent elaboration of Kimmel's formulation. Dykman proposes that habituation and sensitization of the first and second reflexes of a pair determine both the nature and strength of conditioning.

To put formulations of this type to empirical test, habituability, the relative rapidity of habituation, must be capable of experimental manipulation. A number of variables are known to influence the course of habituation, such as intertrial interval and stimulus intensity. Two other variables which may influence habituation, stimulus variability and task requirements, are important both because of their frequent involvement in conditioning procedures and

Experiment 1 was part of the MA thesis of the first author, submitted to the Department of Psychology of the University of South Florida and done under the supervision of the third author. Experiment 2 was done by the second author. because of their potential use in evaluating the influence of habituability on conditioning.

Sokolov (1963) has proposed that making a subject perform a task in response to a stimulus retards habituation of the orienting reaction elicited by the stimulus. The evidence does not support this claim unequivocally. Meyers and Joseph (1968) found no evidence for a differential rate of decline in elicited SCR (skin conductance response) magnitude in human subjects who did not make an RT (reaction time) response, in comparison with those who did. Badia and Harley (1970) also failed to find any evidence for a task effect on rate of SCR habituation in a study of temporal conditioning. In an earlier study, however, Pendergrass and Kimmel (1968) found that making the subject judge the intensity of a loud sound retarded habituation of the SCR to the sound, but only when a variable rather than fixed intertrial interval was used. In the Badia and Harley study, all of the subjects received fixed intertrial intervals. Kimmel, Welch, and Terrant (Kimmel, 1973) found that electrodermal and vasomotor reactions of subjects who judged the intensity of a cool air stimulus habituated at the same rate as those of subjects who did not judge the stimuli. However, when the stimulus was a mild electric shock, judgment of its intensity resulted in sensitization rather than habituation of the electrodermal response, compared with a nonjudgment group, but this effect was not seen in the vasomotor reaction.

In other studies, Germana (1968) found no evidence for a differential rate of SCR habituation depending upon the presence or absence of a task, both between 
and within subjects, while Harding and Punzo (1971) found that SCRs elicited by stimuli associated with an RT task habituated more slowly than SCRs elicited by stimuli that were not associated with a task. The Harding and Punzo results were obtained in within- and between-subject comparisons.

In almost all of these studies, with the exception of that of Badia and Harley (1970), there was either an immediate or eventual difference in SCR magnitude between task and nontask subjects, even though an effect on rate of habituation was not always found. If habituation rate is defined as the persistence (or lack of persistence) of responding to a repeated stimulus (van Olst, 1971), instead of in terms of trial-by-trial changes in response strength, then variables that produce an immediate difference in response magnitude may also produce differences in habituation rate, even though a differential rate of decline in magnitude may not be seen during the first few trials. It should be noted that Sokolov (1963) discussed the effect of task on habituation of the orienting reaction in terms of trials-to-criterion, pointing out that trial-by-trial changes in SCR magnitude in response to task and nontask stimuli are similar. Unfortunately, there are few results available relevant to the effect of task on a trials-to-criterion measure. Van Olst (1971) found that of 120 human subjects who performed an RT task to stimulus offset, elicited SCRs were still present in 117 of these subjects at the end of 25 trials. Although van Olst did not include a nontask control group, he concluded, on the basis of prior experiments, that the average number of trials to criterion had been increased by performance of the task. Ray and Piroch (1976) also reported that an RT task resulted in more frequent elicited SCRs.

There have also been only a few studies of the effect of stimulus variability on habituation. Kimble and Ray (1965) found that habituation of the wiping reflex in the frog was retarded or reversed by varying the location of a cutaneous stimulus within the reflexogeneous zone. A similar effect was noted by Szlep (1964) in a study of the prey-catching response of spiders. However, studies of the effect of tone intensity (Mino \& Minata, 1975) and intertrial interval variability (Gatchel \& Lang, 1974) failed to find an effect of variability on habituation. Since the latter study did not compare presence and absence of variability, it may be that, while habituation is affected by some minimal amount of variation in the intertrial interval, the actual amount of variability above this minimum is not critical. Pendergrass and Kimmel (1968) compared variable and fixed intertrial intervals and found faster habituation with a variable interval, unlike Gatchel and Lang (1974).

The present experiments investigated the effect of task and stimulus variability on both trials to criterion and response magnitude measures. Both SCR and VMR (vasomotor response) were measured in the first study; only SCR was obtained in the second study. It was hypothesized that subjects who perform a task or receive variable stimulation would require more trials to reach a criterion of SCR habituation than subjects not performing the task or not receiving variable stimulation. While overall SCR magnitude was expected to be affected by both task and variability, no definite predictions could be made regarding trial-by-trial magnitude changes.

\section{EXPERIMENT 1}

The first experiment was designed to examine the effects of performing an RT task and receiving variation in the frequency of a pure tone on habituation of the elicited SCR and VMR. The principal measure of habituation was the number of trialsto-criterion, based upon the SCR. Although the main comparisons were between different groups of subjects, an additional group was run to permit a withinsubject assessment of the effect of the task.

\section{Method}

Subjects and design. Fifty female introductory psychology students volunteered to serve as subjects to earn class points. They were randomly assigned to five groups of 10 subjects each, four of the groups resulting from the 2 by 2 factorial combination of the number of stimuli received (one or three) and whether or not an RT task was performed, and the fifth group receiving three stimuli but performing the RT task to only one of them.

Apparatus. The stimuli were $1,000-, 1,200-$, and $1,400-\mathrm{Hz}$ pure tones produced by a General Radio oscillator-amplifying system and delivered via Superex headphones. The intensity of the tones, rated at the phones, was $92 \mathrm{~dB}$ (physical reference). Tone duration was $3.0 \mathrm{sec}$, and the intertrial interval ranged in 2.5 -sec steps from 20 to $40 \mathrm{sec}$, with a mean of 30 . Stimulus presentation and timing were controlled electronically.

The SCR was picked up as a dc resistance change by 1.9-cm zinc-zinc sulfate electrodes in Lucite cups filled with saline jelly. They were attached by rubber straps to the palm and back of the subject's nonpreferred hand. The response was amplified by a Biophysical amplifier and recorded on a Texas Instruments RectiRiter with a paper speed of $15.2 \mathrm{~cm} / \mathrm{min}$. All SCRs are expressed in units of change in log micromhos, measured during the 1-5-sec interval following stimulus onset. Any pen deflection was counted as an SCR in determining the habituation criterion. The VMR was picked up by a photoplethysmograph placed on the distal phalange of the index finger of the nonpreferred hand. The response was amplified and record-

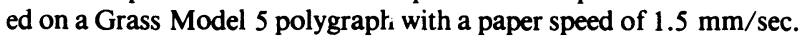
All VMRs are expressed in units of change in pulse magnitude, using the ratio of the four pulses preceding stimulus onset to Pulses 5-8 following onset (Raskin, Kotses, \& Bever, 1969).

Procedure. Data were collected in an IAC sound-attenuated chamber. Prior to her entrance into the chamber, the subject's nonpreferred hand was cleaned with acetone and the electrodermal electrodes were attached. When the subject was seated in the chamber, pick-up leads were connected to the skin electrodes, the photoplethysmograph was attached, the RT switch was placed comfortably in the subject's preferred hand (RT subjects only), and the headphones were put on. The chamber doors were closed, the three different tones were presented for familiarization, and the instructions were then 
read via intercom. The instructions indicated that the subject was to relax and pay attention to the stimuli. Reaction-time subjects were further instructed to press the switch as quickly as possible when the tone came on. Each frequency was presented once for familiarization. The subjects in the group which received three frequencies but reacted to only one were specifically instructed to respond only to the "high," "middle," or "low" one.

Stimuli were presented until two successive SCR nonresponses occurred. All subjects received at least 12 but not more than 60 presentations. Two of the groups received the same frequency on every trial (1S RT and 1S-no RT), the particular frequency used being balanced across subjects. Two of the groups received three different frequencies, in a random order restricted to assure that each of the three would occur four times within each 12 trials (3S-RT and S3-no RT). The fifth group also received all three different frequencies in a random order, permitting each to occur four times within each 12 trials, but were instructed to react only to one of them (3S-1 RT). The frequency to be reacted to was balanced across subjects to this group.

\section{Results}

Trials-to-criterion. Table 1 presents the mean number of trials-to-criterion (plus standard deviations) for all five groups. Subjects who performed the RT task (Groups 1S-RT and 3S-RT) required an average of 39.3 trials to reach the habituation criterion, while those who did not perform the RT task (Groups 1S-no RT and 3S-no RT) required an average of only 10.0 trials. Subjects who received varying stimuli (Groups 3S-no RT, 3S-RT, and 3S-1RT) reached the criterion of habituation in an average of 30.2 trials, while subjects who received invariant stimulation (Groups 1S-no RT and 1S-RT) needed an average of 19.6 trials. Analysis of variance of only the four groups within the 2 by 2 factorial design indicated that the effects of $\mathrm{RT}$ task $(F=39.44$, $\mathrm{df}=1,36)$ and variability $(\mathrm{F}=4.75$, $\mathrm{df}=1,36)$ were significant but that their interaction was not $(\mathrm{F}<1)$. A separate analysis of the trials-to-criterion data of the three groups which received three stimuli showed that they differed significantly $(F=8.69$, df $=2,27$ ). Duncan's new multiple range test indicated that Groups 3S-RT and 3S-1 RT differed significantly from Group 3S-no RT, but no other differences were significant.

SCR magnitude. Table 2 presents the SCR magnitude means during the first 12 trials, averaged in blocks of 3 trials each, for all five groups. ${ }^{1}$ Analysis of variance of these measures for only those groups

Table 1

Means and Standard Deviations of Number of Trials to Criterion in Experiment 1

\begin{tabular}{lcc}
\hline & Mean & Standard Deviation \\
\hline One Stimulus: & & \\
$\quad$ No RT Task & 6.6 & 4.61 \\
$\quad$ RT Task & 32.5 & 17.33 \\
Three Stimuli: & & \\
$\quad$ No RT Task & 13.4 & 7.93 \\
RT Task to All Three & 46.0 & 19.91 \\
RT Task to Only One & 33.1 & 19.44 \\
\hline
\end{tabular}

Table 2

Mean SCR Magnitude Across Four Blocks of Three Trials For All Five Groups in Experiment 1

\begin{tabular}{lcccc}
\hline & \multicolumn{4}{c}{ Trial Blocks } \\
\cline { 2 - 5 } & 1 & 2 & 3 & 4 \\
\hline $\begin{array}{l}\text { One Stimulus: } \\
\quad \text { No RT Task }\end{array}$ & .0213 & .0085 & .0070 & .0060 \\
$\begin{array}{l}\text { Three Stimuli: } \\
\quad \text { No RT Task }\end{array}$ & .0340 & .0204 & .0158 & .0060 \\
$\begin{array}{l}\text { One Stimulus: } \\
\quad \text { RT Task }\end{array}$ & .0386 & .0345 & .0314 & .0277 \\
$\begin{array}{l}\text { Three Stimuli: } \\
\quad \text { RT Task to All Three }\end{array}$ & .0578 & .0796 & .0652 & .0490 \\
$\begin{array}{l}\text { Three Stimuli: } \\
\quad \text { RT Task to Only One }\end{array}$ & .0588 & .0419 & .0414 & .0277 \\
\hline
\end{tabular}

within the 2 by 2 factorial design revealed that the overall SCR magnitudes of subjects who performed the RT task (Groups 1S-RT and 3S-RT) were significantly larger than those of subjects who did not perform the RT task (Groups 1S-no RT and 3Sno $R T)(F=10.56$, df $=1,33)$. The effect of variability was not significant, nor was its interaction with RT task. There was a significant overall effect of trial blocks $(F=3.68$, $\mathrm{df}=3,99)$. However, neither RT task nor variability interacted significantly with trial blocks. A separate analysis of the SCR magnitude data of only the three groups that received three stimuli revealed a significant effect of groups $(\mathrm{F}=4.05, \mathrm{df}=2,24)$ and blocks $(\mathrm{F}=4.33$, df $=$ 3,72 ), but not of the Groups by Block interaction. Duncan's new multiple range test showed that mean SCR magnitude for Group 3S-RT was significantly different from that of Group 3S-no RT, but that Group 3S-1 RT did not differ significantly from either of the other two groups.

Group 3S-1 RT provided a within-subject test of the effect of the RT task. Subjects in this group received a stimulus which was followed by a reaction as well as two stimuli which were not followed by a reaction. Due to the false reactions (reactions to incorrect stimuli) in this group, mean SCR magnitude to stimuli followed by a reaction could not be compared with mean SCR to stimuli not followed by a reaction within each trial block. Overall SCR magnitudes for the first 12 trials for stimuli followed and not followed by a reaction were 0.0489 and 0.0389 , respectively. This difference was statistically significant $(\mathrm{t}=2.33$, $\mathrm{df}=9)$.

VMR magnitude. Table 3 presents mean VMR magnitudes averaged in four blocks of three trials, for the first 12 trials, for all five groups. ${ }^{2}$ Subjects who performed a reaction to each stimulus (Groups 1S-RT and 3S-RT) made larger VMRs than subjects who did not (Groups 1S-no RT and 3Sno RT). Also, VMRs were larger in magnitude in the groups which received variable stimulation (Groups 3S-no RT, 3S-RT, and 3S-1 RT) than in 
Table 3

Mean VMR Magnitude Across Four Blocks of Three Trials For All Five Groups in Experiment 1

\begin{tabular}{lcccc}
\hline & \multicolumn{4}{c}{ Trial Blocks } \\
\cline { 2 - 5 } & 1 & 2 & 3 & 4 \\
\hline $\begin{array}{l}\text { One Stimulus: } \\
\quad \text { No RT Task }\end{array}$ & 1.20 & 1.12 & 1.13 & 1.21 \\
$\begin{array}{l}\text { Three Stimuli: } \\
\quad \text { No RT Task }\end{array}$ & 1.32 & 1.22 & 1.20 & 1.20 \\
$\begin{array}{l}\text { One Stimulus: } \\
\quad \text { RT Task }\end{array}$ & 1.20 & 1.23 & 1.13 & 1.18 \\
$\begin{array}{l}\text { Three Stimuli: } \\
\quad \text { RT Task to All Three }\end{array}$ & 1.43 & 1.46 & 1.31 & 1.34 \\
$\begin{array}{l}\text { Three Stimuli: } \\
\text { RT Task to Only One }\end{array}$ & 1.25 & 1.16 & 1.22 & 1.22 \\
\hline
\end{tabular}

those which received invariant stimulation (Groups 1Sno RT and 1S-RT). Analysis of variance of the VMR magnitude data for those groups within the 2 by 2 factorial design indicated that the effects of $\mathrm{RT}$ task $(\mathrm{F}=11.67, \mathrm{df}=1,33)$ and variability $(\mathrm{F}=5.00$, df $=1,33)$ were significant, but that their interaction was not. There was no trial blocks effect or any interaction with trial blocks. Analysis of variance of only those groups which received three stimuli revealed that only the effect of groups was significant $(F=4.38$, df $=2,24)$. Duncan's new multiple range test showed that the mean VMR for Group 3S-RT differed significantly from those of Groups 3S-no RT and 3S-1 RT; no other comparisons were significant.

RT measures. Mean RT for subjects in Groups 3SRT and 1S-RT were 0.357 and $0.347 \mathrm{sec}$, respectively. This difference was not significant. Mean RT for subjects in Group 3S-1 RT was $1.980 \mathrm{sec}$, i.e., much slower. This difference between the RT means of subjects reacting to all stimuli and subjects reacting to only some stimuli agrees with classical findings concerning simple vs. disjunctive RT.

\section{Discussion}

When measured in terms of trials to criterion, SCR habituation was markedly retarded by the performance of the RT task and somewhat less retarded by variability of stimulation. Differential trial-by-trial changes in SCR magnitude were not seen in the different groups, even though reliable overall SCR magnitude differences were. The overall magnitude effect of RT task was also found in a within-subject comparison, although in that comparison it was impossible to assess trial-by-trial changes in magnitude within subjects.

The greater sensitivity of the trials-to-criterion measures to the influence of the task and variability factors has several possible causes. First, and most likely, the considerable variability of individual SCR habituation functions may simply have produced so large a Subjects by Trial Blocks error estimate that a "true" differential habituation rate (in terms of magnitude decline) could not be detected without a much larger sample of subjects. Second, if the "true" habituation function is of the steeply arched, curvilinear type that is commonly obtained, large differences in trials-to-criterion scores might be obtained between two essentially parallel functions which differ only slightly in absolute level. If the SCR level of the task function equaled that of the nontask function plus an additive constant, a Groups by Trials interaction would not be seen in the earlier trials (not until the nontask function reached zero). However, a trials-to-criterion difference could be seen. Although the last conjecture appears to fit the results of several earlier studies (Germana, 1968; Meyers \& Joseph, 1968), changes in SCR magnitude across trial blocks did not appear to be parallel in the present study.

It is interesting to note that, although no significant VMR habituation was observed, overall VMR magnitude was significantly affected by both task and variability. Also, the effects of task and variability on VMR magnitude were similar, while the effect of task on the SCR trials-to-criterion measures was much more potent than that of variability.

\section{EXPERIMENT 2}

One possible explanation for the effect of the RT task on the SCR habituation observed in Experiment 1 is that the muscular act itself may maintain larger and more persistent SCRs either through proprioceptive feedback or effort. It is known that SCRs can be elicited by voluntary movements, and their magnitudes have been shown to depend upon the amount of tension exerted on a hand dynamometer (Epstein, Boudreau, \& Kling, 1975). Although there were many occasions in Experiment 1 when subjects made a skeletal reaction without making an autonomic one, it is possible that autonomic reactions elicited by contraction may behave as variably as when they are elicited by other stimuli. Indeed, to the extent that skeletal reactions may vary from occasion to occasion in their character and vigor, their proprioceptive consequences may provide variable, as distinguished from invariant, eliciting stimuli, a condition which might directly foster slower habituation.

Beyond a direct stimulational effect of the RT task, the set to perform skeletal reactions, which was present in all of the groups performing the RT task in Experiment 1, may itself retard habituation of elicited autonomic components of the orienting reactions. Comparison of SCRs elicited on trials on which no skeletal reaction was performed in Experiment 1 , between groups which received three stimuli and made skeletal reactions to either none of them or to only one of them (3S-no RT vs. 3S-1RT), 
indicated that the mean SCR when no skeletal reaction was made was larger when a set to react to one of the stimuli was present than when no RT set was present $(\mathrm{t}=2.11, \mathrm{df}=18)$. This comparison excluded trials on which false reactions occurred, for obvious reasons. Because these possible sources of retardation of habituation were not explicitly or fully dealt with in Experiment 1, it was decided to examine them more directly in Experiment 2.

The purpose of Experiment 2 was to assess the effect of an RT task on SCR habituation when skeletal contraction is totally eliminated as a possible source of elicited SCRs. This was achieved by having subjects react to stimulus offset and measuring the SCR elicited by stimulus onset, well before the occurrence of any contraction. In addition, the effect of stimulus duration variability on SCR habituation was examined. It was expected that subjects who performed an RT task to stimulus offset, or who received variable stimulus durations, would require a greater number of trials to reach a habituation criterion and would make larger SCRs than control subjects who did not perform the task or did not receive variable durations. Since the subjects were explicitly instructed to withhold the skeletal response until the stimulus terminated, variability of duration was obviously a task-related factor, unlike the task-independent factor of frequency variability in Experiment 1.

\section{Method}

Subjects and design. Forty-eight female introductory psychology students at the University of South Florida volunteered to serve as subjects to earn class points. They were randomly assigned in equal numbers to the four groups resulting from the factorial combination of RT task (reaction to offset vs. no reaction) and variability of duration (three different stimulus durations vs. constant duration).

Apparatus. The stimulus was a $1,000-\mathrm{Hz}$ pure tone, 4,6 , or $8 \mathrm{sec}$ in duration and delivered via Superex headphones. The intensity of the tone, rated at the phones, was $42 \mathrm{~dB}$ (physical reference). Tones were presented in 5-sec steps with a 20 - to 60-sec intertrial interval with a mean of $40 \mathrm{sec}$. SCRs were picked up, amplified, and recorded as in Experiment 1, and stimuli were produced and generated likewise.

Procedure. Preexperimental procedure was the same as in Experiment 1, except that the instructions were modified to require that the subject respond as quickly as possible following stimulus offset, but not before. As in Experiment 1, all subjects received at least 12 and not more than 60 trials, and the habituation criterion was two successive nonresponses, with the SCR being defined as in Experiment 1 .

Subjects in the constant-duration groups (1D-RT and 1D-no RT) received only one duration of the stimulus, the three different constant durations being balanced across subjects. Subjects in the variable duration groups (3D-RT and 3D-no RT) received all three durations in a random order, which assured that each duration occurred four times within each 12 trials.

\section{Results}

Table 4 presents the mean number of trials to criterion (plus standard deviations) for all four groups. Subjects who performed the RT task to
Table 4

Means and Standard Deviation of Number of Trials to Criterion in Experiment 2

One Duration

Three Durations

$\begin{array}{lll}\text { No RT Task } & & \\ \text { Mean } & 7.4 & 8.3 \\ \text { SD } & 3.71 & 5.07 \\ \text { RT Task } & & \\ \text { Mean } & 38.6 & 21.2 \\ \text { SD } & 20.48 & 10.37\end{array}$

stimulus offset required an average of 29.9 trials to reach the criterion, while those who did not perform the task required an average of 7.8 trials. Although there was an overall difference in number of trials to criterion between subjects receiving variable durations of stimuli and those receiving a constant stimulus duration, inspection of Table 4 shows that this difference was confined entirely to the RT groups. The group which performed the RT task and had variable durations of stimuli required an average of 17.4 fewer trials to reach criterion than those who did not perform the task but had variable durations of stimuli. Analysis of variance of the trials-tocriterion data showed that the effects of RT task $(F=37.59$, $d f=1,44)$ and variability $(F=5.29$, $\mathrm{df}=1,44)$, and the interaction of task and variability $(\mathrm{F}=6.53, \mathrm{df}=1,44)$, were all significant.

Mean SCR magnitude, averaged in blocks of 3 trials across the first 12 trials for all groups, is shown in Table 5. Although the means were ordered in the same way as those of the trials-to-criterion data, analysis of variance indicated that only the effect of task was significant $(\mathrm{F}=14.67$, df $=1,44)$. Analysis of the SCR magnitude data over the four blocks of 3 trials revealed that only the effect of blocks was significant $(F=8.99$, $\mathrm{df}=3.132)$, indicating that, while overall habituation occurred, there were no significant differences in the amount of decline in SCR magnitude among the four groups. Thus, SCR habituation rate could be said to have been affected by the RT task only in terms of the trials-to-criterion data, since changes in SCR magnitude over trial blocks did not appear to differ for the groups that performed the task and those that did not. The subjects who performed the task mainly made larger overall responses, indicating that the differences in

Table 5

Mean SCR Magnitude Across Four Blocks of Three Trials Each for All Four Groups in Experiment 2.

\begin{tabular}{lccccc}
\hline & & \multicolumn{4}{c}{ Trial Blocks } \\
\cline { 3 - 6 } & & 1 & 2 & 3 & 4 \\
\hline \multirow{2}{*}{ No RT } & 1D & .0253 & .0039 & .0036 & .0013 \\
& 3D & .0157 & .0131 & .0084 & .0011 \\
\multirow{2}{*}{ RT } & 1D & .0550 & .0405 & .0381 & .0288 \\
& 3D & .0508 & .0324 & .0311 & .0288 \\
\hline
\end{tabular}


number of trials to criterion may have been due to level differences rather than to differential SCR magnitude functions.

Average RT was slower in subjects who received variable stimulus durations than in those who did not, but this difference was not statistically significant.

\section{Discussion}

The results of Experiment 2 establish that the effect of the RT task on SCR habituation in both experiments cannot be attributed simply to contractionelicited SCRs. Subjects who performed the RT task to the offset of the tone showed greater persistence in responding to its onset, before contraction occurred, than was shown by subjects who did not perform this task. This finding is in agreement with previous evidence that it is not simply the performance of a motor act, itself, that influences orienting reactions to stimuli which are presented in the same situation as the act, but that it is the direct association of the act with the stimulus. For example, Harding and Punzo (1971) and Harding, Stevens, and Marston (1973) showed that subjects who are instructed to perform motor acts unconnected with the occurrence of a stimulus make smaller SCRs both to the stimulus and to the motor act than do subjects who perform the motor act in response to the stimulus.

Variability of the duration of the tone, unlike the frequency variability of Experiment 1, did not itself produce either a larger number of trials to criterion or larger SCR magnitude. Duration variability did, however, interact with the offset RT task, at least in terms of the trials-to-criterion data. Subjects who performed the RT task and received varying tone durations took fewer trials to reach the habituation criterion than did subjects who performed the task but had a constant tone duration. This "subtractive" interaction (i.e., the effect of task plus the effect of variability was smaller than the effect of task alone) suggests that either task difficulty or response uncertainty, or their combination, may have altered the usual task effect. Task difficulty is not uniformly positively, related to the magnitude of peripheral autonomic changes. Obrist (1976) found that a moderately difficult task produced a larger increase in systolic blood pressure than either a very easy or a very difficult task. It may be that, at high levels of task difficulty, sympathetic activity is protectively attenuated in order to minimize disruption associated with excessively high levels of activation. Similar results have been found by Harley (1973), in a study of temporal conditioning of the SCR. Harley's subjects were instructed to anticipate stimulus onset, and they made larger intertrial responses when a fixed intertrial interval was used than when a variable one was. The former of Harley's tasks was obviously less difficult than the latter.
The task of responding as quickly as possible to the offset of a stimulus the duration of which varied unpredictably from 4 to 6 to $8 \mathrm{sec}$ was probably more difficult than that of responding to the offset of a stimulus with a constant duration of 4,6 , or $8 \mathrm{sec}$. But, without explicit study of the effect of difficulty unconfounded with variability of duration, it cannot be concluded that task difficulty, per se, was responsible for the more rapid habituation observed in Group 3D-RT as compared to Group 1DRT. "Difficulty" in this context, of course, refers to the relative ease in performing the task that the subject was instructed to perform-to respond as quickly as possible to the offset of a stimulus, but not before its offset. If the duration of the stimulus is analogous to the foreperiod in a standard RT paradigm, it is likely that the longer two durations (6 and $8 \mathrm{sec}$ ) made the offset task more difficult because of their absolute length as well as via uncertainty in the variable condition. Only the 4-sec duration can be considered even close to the optimal RT foreperiod (Woodrow, 1914). To check on this possibility, twelve additional subjects were run in the constant duration condition, following initial data reduction, bringing the size of the 4-, 6-, and 8 -sec constant-duration subgroups up to eight. The mean number of trials to the SCR habituation criterion in these three subgroups were $39.9,36.6$, and $23.8(4,6$, and $8 \mathrm{sec}$, respectively), not significantly different but inversely related to duration-difficulty. Thus, although no firm conclusion on this point may be stated, it is possible that both difficulty via longer durations and uncertainty via variable durations contributed to the reduced persistence seen in the variable-duration $\mathrm{RT}$ group as compared to the constant duration RT group. Of course, both RT groups showed greater persistence than the no-task groups.

\section{GENERAL DISCUSSION}

Although requiring that subjects perform a task in connection with a stimulus strongly retarded SCR habituation in terms of trials to criterion, more difficult tasks appeared to result in less retardation of habituation than did somewhat less difficult tasks. In Experiment 1, the degree to which the onset RT task retarded habituation (compared to no task) was less in subjects who performed the more difficult disjunctive RT task than in subjects who performed the easier RT task to all three stimuli. Likewise, in Experiment 2, the retardation produced by performing an offset RT task (compared to no task) was less when difficulty was increased by presenting variable stimulus durations than when stimulus duration was constant.

The greater difficulty of the disjunctive RT condition of Experiment 1 was manifested in an average RT of $1.98 \mathrm{sec}$, which was not only much slower 
than the average RT in the other group that had three tone frequencies but reacted to all of them but, also, meant that the actual skeletal reaction occurred at a time after the autonomic reaction to stimulus onset had already begun. Similarly, in Experiment 2, increased difficulty associated with variable and/or longer stimulus durations was also focused at a temporal point well beyond stimulus onset. Yet, in both experiments, the SCRs which entered into the trials-to-criterion data and which determined the criterion of habituation were responses elicited by stimulus onset. In view of this, it is possible that increased persistence in postonset reactivity (or offset reactivity) may have a negative effect on persistence of onset reactivity. If the SCR which occurs at the moment of execution of the offset RT task of Experiment 2 aids in the execution of the task in some fashion, it might be adaptively economical for SCRs which immediately precede such "execute" SCRs to be attenuated. Likewise, in the disjunctive RT condition in Experiment 1, with subjects reacting skeletally well after the autonomic reaction to stimulus onset has been initiated, a similar mechanism for adaptive attenuation of onset SCRs could have been operating.

This kind of attenuation of onset reactivity could readily be accomplished by a negative feedback mechanism. Although increased persistence of postonset reactivity may have negatively influenced onset reactivity in these cases, it would be neither necessary nor desirable for increased persistence of onset reactivity to have a similar negative effect on offset reactivity. After all, a postonset reaction or an offset reaction could not possibly interfere with the preceding onset reaction. Instead, factors enhancing onset reactivity should augment offset reactivity also. The sign of the product of the functions describing these positive and negative relationships is negative, satisfying the formal requirements for the existence of negative feedback.

The potential utility of this approach to comprehending the difference in SCR habituation between two groups who performed the offset RT task and received either variable or constant stimulus durations requires a demonstration of differential SCR reactivity at stimulus offset in Experiment 2. Although the mean offset SCR magnitudes of these two groups did not differ significantly over the first 12 trials, an interesting trials-to-criterion effect occurred. Offset SCRs reached the habituation criterion before onset SCRs did in 11 of the 12 subjects in the 1D-RT group, but this was true for only four of the subjects in the 3D-RT group. This difference was significant (chi square $=8.70, p<.01$ ), indicating that offset SCRs were more persistent relative to onset SCRs in the variable duration group as compared with the constant duration group. Comparison of absolute fre- quencies of offset SCRs between these two groups was not possible, since the session was terminated when the subject's onset SCR habituated, whether or not the offset SCR had. However, the negative feedback model does not require that absolute SCR frequencies in the 1D-RT and 3D-RT groups differ. If both groups begin the session with similar onset reactivities and one group's offset reactivity remains more persistent or increases relative to the other, after a few trials the latter group's onset reactivity should reduce and the number of trials-to-criterion for the onset reaction should be reduced accordingly. If the negative feedback is of the "amplifying" type, onset reactivity will reduce more than offset reactivity increases. If frequency of SCRs elicited by stimulus offset is a positive function of both onset and offset reactivity, offset SCR frequency might be reduced, rather than increased, when offset reactivity increases, because of the greater decrease in onset reactivity.

The effect of the task itself probably influences onset reactivity only directly. In Experiment 1, making the subjects perform a response to the stimulus produced greater onset reactivity than was present without the task. If the positive influence of onset reactivity on postonset reactivity is small in relation to the negative influence of postonset reactivity on onset reactivity the possibility that negative feedback would then reduce onset reactivity is negligible. In Experiment 2, on the other hand, the task requirement probably increased both onset and offset reactivity. The group with the largest offset reactivity, Group 3D-RT, had its onset reactivity reduced by the negative feedback from offset to onset reactivity and, accordingly, required fewer trials to criterion than Group 1D-RT. Both groups, however, were more persistent than the nontask groups because of the overall increase in onset reactivity resulting from the task. Likewise in Experiment 1, task subjects showed greater persistence in responding due to increased onset reactivity (postonset reactivity having no differential effects in this case, except in the disjunctive RT condition, and effectively cancelling out).

In summary, it is suggested that manipulations which tend to increase reactivity after stimulus onset have a subtractive effect on reactivity at onset. Reactivity at or near offset can be increased by manipulations which increase the latency of a task reaction or which increase the task difficulty at offset. An example of the first kind of manipulation was the disjunctive RT task in Experiment 1 , while the variable-stimulus duration-response to offset task in Experiment 2 is an example of the second kind of manipulation. Both manipulations appear to reduce the overall habituation-retarding effect of task, which itself was probably due to the direct effect of the task on the persistence of onset reactivity. 


\section{REFERENCES}

Badia, P.. \& Harley, J. P. Habituation of temporal conditioning as related to shock intensity and its judgment. Journal of Experimental Psychology, 1970, 84. 534-536.

Dy kman, R. A. Conditioning as sensitization. The Pavlovian Journal of Biological Science, 1976, 11, 24-36.

Epstein. S.. Boudreau, L., \& King, S. Magnitude of the heart rate and electrodermal response as a function of stimulus input. motor output. and their interaction. Psychophysiology, 1975, 12. 15-24.

Gatchel, R. J., \& LANG, P. J. Effects of interstimulus interval length and variability on habituation of autonomic components of the orienting response. Journal of Experimental Psychology, 1974. 103. 802-804.

Germana. J. Response characteristics and the orienting reflex. Journal of Experimental Psychology, 1968, 78. 610-616.

Harding. G.. \& Punzo, F. Response uncertainty and skin conductance. Journal of Experimental Psychology, 1971, 88. $265-272$.

Harding, G.. Stevens, E. E., \& Marston, P. T. Rate of information processing and skin resistance. Journal of Experimental Psychology, 1973, 99, 306-313.

HARLEY. J. P. Temporal conditioning as a function of instructions and intertrial interval. Journal of Experimental Psychology, 1973. 100. 179-184.

Kimble, D. P., \& RAY, R. S. Reflex habituation and potentiation in Rana pipiens. Animal Behavior, 1965, 13, 530-533.

KIMMEL. H. D. Habituation, habituability and conditioning. In H. V. Peeke \& M. J. Herz (Eds.), Habituation (Vol. 1) Behavioral studies. New York: Academic Press, 1973. Pp. 219-238.

KozaK. W..\& Westerman, R. A. Basic patterns of plastic change in the mammalian nervous system. Symposium of the Society for Experimental Biology, 1966, 20, 509-544.

MEYers. W. J.. \& Joseph, L. J. Response speed as related to CS prefamiliarization and GSR responsivity. Journal of Experimental Psychology, 1968, 78, 375-381.

Mino. T..\& MiYata, Y. Effects of the range of stimulus-variation upon habituation of the orienting response. Psychophysiology, $1975,12,136-140$.

ObRIST, P. A. The cardiovascular-behavioral interaction as it appears today. Psychophysiology, 1976, 13, 95-107.

Pendergrass, V. E.. \& Kimmel, H. D. UCR diminution in tem- poral conditioning and habituation. Journal of Experimental Psychology, 1968, 77, 1-6.

Raskin, D. C., Kotses, H., \& Bever, J. Cephalic vasomotor and heart rate measures of orienting and defensive reflexes. Psychophysiology, 1969, 6, 149-159.

RAY, R. L., \& PIROCH, J. F. Orienting responses to a change in stimulus significance. Bulletin of the Psychonomic Society, 1976, 8. 82-84.

Razran, G. Mind in evolution. Boston: Houghton, 1971.

Sokolov, Y. N. Perception and the conditioned reflex. Oxford: Pergamon, 1963.

SzLEP, R. Change in the response of spiders to repeated web vibrations. Behaviour, 1964, 23, 203-238.

Thompson, R. F., \& Spencer, W. A. Habituation: A model phenomenon for the study of neuronal substances of behavior. Psychological Review, 1966, 173, 16-43.

THORPE, W. H. Learning and instinct in animals. London: Methune, 1956.

VAN OLST, E. H. The orienting reflex. Netherlands: Mouton, 1971. Woodrow, H. Measurement of attention. Psychological Monographs, 1914, 17(Whole No. 76).

\section{NOTES}

1. An examination of SCR magnitude on Trial 1 showed a significant initial difference between groups. Inspection of the data revealed that the source of this was that three subjects in Group 3S-RT made very large first responses. Since this initial difference produced an apparent "effect" of frequency variability before more than one stimulus was actually presented, the magnitude data from these subjects were discarded. Data from these subjects were used, however, in the evaluation of trials to criterion since initial SCR magnitude and trials to criterion were unrelated $(r=.09)$. There was no first-trial difference between Groups 1S-RT and 1S-no RT.

2. Because of equipment difficulties, VMR data were not available for two subjects in Groups 3S-RT and for one subject each in Groups 1S-no RT and 3S-1RT.

(Received for publication August 5, 1976; revision accepted December $13,1976$. 